\title{
ORIGINAL ARTICLE \\ Prevalence of androgen deficiency in chronic spinal cord injury patients suffering from erectile dysfunction
}

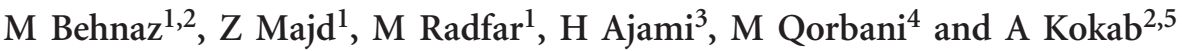

Study design: Retrospective cross-sectional study.

Objectives: To estimate the prevalence of biochemical androgen deficiency in Iranian spinal cord injured men suffering from erectile dysfunction (ED) and to determine factors related to this problem.

Setting: An andrology clinic of a teaching hospital in Tehran.

Methods: Men, 18 years of age and older, with chronic ( $>2$ years) spinal cord injury (SCI) suffering from ED were recruited. Demographic data, hormonal and lipid levels, injury variables and drug history were obtained from the medical records. The relationships between biochemical androgen deficiency (unequivocally low serum testosterone levels) and patient characteristics, injury variables and laboratory data were determined.

Results: $\quad$ Out of 319 patients, 32.6\% had total testosterone deficiency and $29.1 \%$ had biochemical androgen deficiency. Of those with biochemical androgen deficiency, 93.5 and $85.7 \%$ had luteinizing hormone (LH) and follicle stimulating hormone (FSH) within or below the normal ranges, respectively. Opioid use, triglyceride (TG) and total cholesterol (TC) were associated with biochemical androgen deficiency. Significant correlations between total testosterone level and sex hormone binding globulin $(r=0.3, P<0.001)$, $\mathrm{LH}(r=0.2, P=0.02)$, TC $(r=-0.1, P=0.04)$ and TG $(r=-0.3, P<0.001)$ were found.

Conclusion: A substantial proportion of our patients with chronic SCl and ED had biochemical androgen deficiency. Opioid use, TG and TC levels were associated with biochemical androgen deficiency in our studied population. Standard screening of androgen deficiency and testosterone replacement therapy are recommended in men with chronic SCI suffering from ED.

Sponsorship: Tehran University of Medical Sciences.

Spinal Cord (2017) 55, 1061-1065; doi:10.1038/sc.2017.73; published online 20 June 2017

\section{INTRODUCTION}

Spinal cord injury (SCI) is related to variety of problems in human body systems. Previous studies have shown high prevalence of cardiovascular, musculoskeletal, urological, reproductive and endocrine complications in patients suffering from SCI. Two of the most important concerns in spinal cord injured men are erectile dysfunction (ED) and alterations in the secretion of hormones such as testosterone and gonadotropins. ${ }^{1-4}$ Testosterone as a principle male sex hormone has several important functions. Decline in serum testosterone concentration has negative effects on the body, from bone and muscle loss, to sexual and reproductive dysfunctions, to psychological and mental disorders. ${ }^{5,6}$

Androgen deficiency is a clinical syndrome with typical signs and symptoms and unequivocally low serum testosterone levels, according to the Endocrine Society clinical practice guidelines. ${ }^{7}$ Common clinical features in men with SCI are similar to hypogonadal men. It suggests that these health problems could be attributed to androgen deficiency which may play a crucial role in the quality of life and well-being of SCI patients. Taken together, it seems that identification of androgen deficiency as a treatable co-morbidity is important in this population., ${ }^{1,8}$
In recent years, some studies have reported high prevalence of testosterone deficiency in men following SCI, while others have not found this abnormality. Given that acute physical distress and systemic diseases affect testosterone level, ${ }^{9}$ high incidence of testosterone deficiency is expected following acute SCI. However, the effect of chronic SCI on serum testosterone level is still controversial. The reason may be lack of studies with adequate sample size and methodological concordance. ${ }^{10,11}$

In this study we aimed to determine the prevalence of biochemical androgen deficiency in a large population of Iranian men with chronic SCI suffering from ED. The secondary goal of this study was to investigate the relationship between this problem-biochemical androgen deficiency-and patient characteristics, injury variables and laboratory data.

\section{METHODS}

This retrospective and cross-sectional study was carried out in Shafa Research Center, Khatam Al-Anbia hospital, Tehran, Iran from January 2013 to March 2015. The protocol was approved by the institutional review board and ethics committee. Three hundred nineteen men with established SCI attending an andrology clinic were recruited into the study. Men, 18 years of age and older,

${ }^{1}$ Faculty of Pharmacy, Department of Clinical Pharmacy, Tehran University of Medical Sciences, Tehran, Iran; ${ }^{2}$ Shafa Neuroscience Research Center, Khatam Al-Anbia Hospital, Tehran, Iran; ${ }^{3}$ Department of Obstetrics, Treatment Deputy of Alborz University of Medical Sciences, Karaj, Iran; ${ }^{4}$ Non-Communicable Disease Research Center, Alborz University of Medical Sciences, Karaj, Iran and ${ }^{5}$ Department of Urology, Royal Hallamshire Hospital, University of Sheffield, Sheffield, UK

Correspondence: Dr M Radfar, Faculty of Pharmacy, Department of Clinical Pharmacy, Tehran University of Medical Sciences, Enghelab Square,16 Azar Ave, PO Box: 14155/6451, Tehran 1417614411, Iran.

E-mail: radfarma@tums.ac.ir

Received 28 November 2016; revised 29 May 2017; accepted 18 May 2017; published online 20 June 2017 
with chronic SCI ( $>2$ years since injury) suffering from ED were included in our study. Patients were excluded if had prostate cancer, pituitary microadenoma, chronic liver or kidney disease, respiratory failure, coronary artery disease, history of traumatic genitalia injury and history of taking medications that may alter testosterone levels, including anabolic steroids, corticosteroids, finasteride, spironolactone, ketoconazole, digoxin, verapamil, carbamazepine, valproic acid and phenytoin. As our participants used low potency and short-acting opioid analgesics (for example, codeine and tramadol) on an as needed basis, we did not consider opioid use as an exclusion criterion.

Patient characteristics, date and neurologic level of injury, American Spinal Injury Association Impairment Scale (AIS) grade, drug history and laboratory values were obtained from the medical records. Laboratory values included total testosterone (TT), sex hormone binding globulin (SHBG), follicle stimulating hormone (FSH), luteinizing hormone (LH), prolactin (PRL), triglyceride (TG), total cholesterol (TC) and albumin.

Neurological level and completeness of the lesion were defined by using International Standards for Neurological Classification of SCI. ${ }^{12}$ Moreover, ED was evaluated by an andrologist according to physical examination and International Index of Erectile Function (IIEF). ${ }^{13}$

All blood samples were drawn in a fasting state between 0800 and 1000 hours, measured by the same laboratory. Total testosterone, LH, FSH and PRL were measured by enzyme-linked fluorometric assay technique according to the manufacturer's guidelines on the Tosoh AIA system analyzer (Tosoh Bioscience N.V., Tessenderlo, Belgium). The serum SHBG level was determined using enzyme-linked immunosorbent assay kit (IBL international, Hamburg, Germany). The lower limit of detection for testosterone, LH, FSH, PRL and SHBG was $0.2 \mathrm{nmoll}^{-1}, 0.2 \mathrm{I} \mathrm{Ul}^{-1}, 1.0 \mathrm{I} \mathrm{Ul}^{-1}, 44 \mathrm{pmoll}^{-1}$ and $0.77 \mathrm{nmol}^{-1}$, respectively. Triglyceride and TC were assessed by enzymatic photometric technique (kit: Parsazmun Company, Tehran, Iran). Serum albumin was measured by bromocresol green method using a commercial kit (Parsazmun Company).

According to Endocrine Society guidelines, biochemical androgen deficiency was defined as TT levels lower than $10.4 \mathrm{nmol}^{-1}$. Alternatively, FT levels $<0.225 \mathrm{nmol}^{-1}$ were used as a criteria for biochemical androgen deficiency in patients in whom TT levels were insufficient for diagnosis-patients with SHBG abnormality or TT levels near the lower limit of normal. ${ }^{7}, 14$ Vermeulen formula was used to calculate FT in these patients. ${ }^{15}$ The formula is available on the ISSAM Web site (www.issam.ch/freetesto.htm).

High PRL levels were determined as stated in the Endocrine Society and Pituitary Society guidelines. ${ }^{16,17}$ Interpretation of other laboratory data was based on manufactures' instruction. Reference ranges for SHBG, LH and

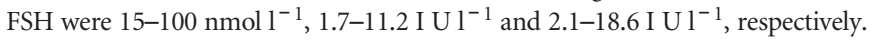

We certify that all applicable institutional and governmental regulations concerning the ethical use of human volunteers were followed during the course of this research.

\section{Statistical analysis}

Statistical analysis was performed using SPSS software version 16.0. Normal distribution of continuous variables was assessed using Kolmogorov-Smirnov test. Continuous variables with normal distribution were described as mean and s.d. Variables with lack of normal distribution were expressed as median and interquartile range (IQR).The mean difference of normally distributed parameters in studied groups was assessed using independent sample $t$-test, while Mann-Whitney $U$ test was used to analyze the significance of the difference between nonparametric variables. Categorical data were shown as number (percentage). Associations between categorical data and biochemical androgen deficiency were examined by using $\chi^{2}$ analysis. Spearman's correlation test was performed to investigate the correlations. The association of demographic and clinical characteristics with risk of biochemical androgen deficiency was assessed using two models of logistic regression analysis. Model 1 was crude association between each independent variables and biochemical androgen deficiency (univariate model), whereas in Model 2 this association was adjusted for age, duration of SCI, level of injury, extent of injury, smoking and opioid usage (multivariate model). The results were described as odds ratio (OR) and 95\% confidence interval (CI). A $P$-value $<0.05$ was considered statistically significant.
Table 1 Sociodemographic and disease characteristics of participants in studied groups

\begin{tabular}{|c|c|c|c|}
\hline \multirow[t]{2}{*}{ Variables } & \multicolumn{2}{|c|}{ Biochemical androgen deficiency } & \multirow[t]{2}{*}{ P-value } \\
\hline & Yes $(n=93)$ & No $(n=226)$ & \\
\hline Age (year) ${ }^{\mathrm{a}}$ & $47.4(6.7)$ & $47.1(7.8)$ & 0.707 \\
\hline $\mathrm{SCl}$ duration (year) $)^{\mathrm{b}}$ & $27.0(25.0-29.0)$ & $26.0(24.0-29.0)$ & 0.099 \\
\hline \multicolumn{4}{|l|}{ Marital status ${ }^{c}$} \\
\hline Married & 89 (95.7) & $213(94.2)$ & 0.869 \\
\hline Single & $3(3.2)$ & $10(4.4)$ & \\
\hline Divorced & $1(1.1)$ & $3(1.3)$ & \\
\hline \multicolumn{4}{|l|}{ Smokingc } \\
\hline Yes & $31(33.3)$ & $55(24.4)$ & 0.105 \\
\hline No & $62(66.7)$ & $170(75.6)$ & \\
\hline \multicolumn{4}{|l|}{ Opioid use ${ }^{c}$} \\
\hline Yes & $16(17.2)$ & $21(9.3)$ & 0.045 \\
\hline No & $77(82.8)$ & $205(90.7)$ & \\
\hline \multicolumn{4}{|l|}{ Level of injuryc } \\
\hline Cervical & 12 (13.0) & $31(13.8)$ & 0.204 \\
\hline Thoracic & $54(58.7)$ & $150(67.0)$ & \\
\hline Lumbosacral & $26(28.3)$ & $43(19.2)$ & \\
\hline \multicolumn{4}{|l|}{ Extent of injuryc } \\
\hline ASIA score A (complete) & $45(50.0)$ & $124(57.4)$ & 0.235 \\
\hline ASIA score B-D (incomplete) & $45(50.0)$ & $92(42.6)$ & \\
\hline \multicolumn{4}{|l|}{ Wheelchair bound } \\
\hline Yes & $69(82.1)$ & $191(89.3)$ & 0.098 \\
\hline No & 15 (17.9) & $23(10.7)$ & \\
\hline
\end{tabular}

Abbreviations: ASIA, American spinal injury association; IQR, interquartile range; $\mathrm{SCI}$, spinal cord injury.

aAge was expressed as mean (s.d.).

bDuration of $\mathrm{SCl}$ was expressed as median (IQR).

${ }^{\mathrm{c} C a t e g o r i c a l ~ v a r i a b l e s ~ w e r e ~ e x p r e s s e d ~ a s ~ n u m b e r ~(p e r c e n t a g e) . ~}$

\section{RESULTS}

The mean (s.d.) age of our study population was 47.2 (7.5) years with range of 24-77 years. The median (IQR) duration of their SCI was 26.0 (24.0-29.0) years with range of 2-33 years. Biochemical androgen deficiency was confirmed in 93 patients (prevalence: $29.1 \%$, 95\% CI: 24.2-34.5). Low TT level was found in 104 patients (prevalence: $32.6 \%$, 95\% CI: 27.5-38.1). The median (IQR) of TT was 12.4 $(9.0-16.5) \mathrm{nmoll}^{-1}$ in the studied population.

Sex hormone binding globulin level was low in 48 (15.0\%) patients. None of the participants had high SHBG level. In participants with low SHBG levels, 26 (54.1\%) had normal TT and FT levels, $13(27.1 \%)$ had low TT and normal FT levels and $9(18.7 \%)$ had low TT and FT levels. TT level near the lower limit of normal was detected in $17(5.3 \%)$ patients. Of those, 9 (52.9\%) had normal TT and FT levels, 5 (29.4\%) had normal TT level and low FT level and $3(17.6 \%)$ had low TT level and normal FT level.

Among patients with biochemical androgen deficiency, 87 (93.5) and $72(85.7 \%)$ had low or normal LH and FSH, respectively. Six (6.5\%) had high LH and 12 (14.3\%) had high FSH levels in biochemical androgen deficient group. High PRL level was observed in one subject who had also biochemical androgen deficiency.

Tables 1 and 2 exhibit sociodemographic and disease characteristics of participants and laboratory data in biochemical androgen deficient 
Table 2 Laboratory data of patients in studied groups

\begin{tabular}{|c|c|c|c|}
\hline \multirow[t]{2}{*}{ Variables } & \multicolumn{2}{|c|}{ Biochemical androgen deficiency } & \multirow[t]{2}{*}{ P-value } \\
\hline & Yes $(n=93)$ & No $(n=226)$ & \\
\hline Total testosterone $\left(\mathrm{nmol} \mathrm{I} \mathrm{I}^{-1}\right)^{\mathrm{a}}$ & $7.3(6.1-9.0)$ & $14.2(12.1-18.4)$ & $<0.001$ \\
\hline SHBG $\left(n m o l I^{-1}\right)^{a}$ & $26.3(20.5-33.9)$ & $25.0(18.0-37.7)$ & 0.827 \\
\hline LH $\left(I U I^{-1}\right)^{a}$ & $3.6(2.1-5.5)$ & $3.9(2.6-5.9)$ & 0.208 \\
\hline $\mathrm{FSH}\left(\mathrm{IU} \mathrm{I}^{-1}\right)^{\mathrm{a}}$ & $3.8(2.5-6.7)$ & $4.3(2.7-6.9)$ & 0.569 \\
\hline Prolactin $\left(\mathrm{pmol} \mathrm{I}^{-1}\right)^{\mathrm{a}}$ & $358.9(251.7-437.7)$ & $361.1(252.5-498.9)$ & 0.587 \\
\hline Triglyceride (mg dl-1)a & $152.0(103.0-216.0)$ & $126.0(95.0-191.0)$ & 0.022 \\
\hline Total cholesterol $\left(\mathrm{mg} \mathrm{dl}^{-1}\right)^{\mathrm{b}}$ & $186.6(37.1)$ & $175.9(38.2)$ & 0.027 \\
\hline
\end{tabular}

Abbreviations: FSH, follicle stimulating hormone; IQR, interquartile range; LH, luteinizing hormone; SHBG, sex hormone binding globulin.

aData were presented as median (IQR).

Data were presented as mean (s.d.).

and normal patients, respectively. There were 37 (11.6\%) subjects who took opioids in our study. Opioid use was significantly higher in patients with biochemical androgen deficiency than normal group $(P<0.05)$.

Total cholesterol and TG levels were significantly higher in biochemical androgen deficient men $\left(P^{\prime} s<0.05\right)$. None of the patients reported alcohol consumption in their history.

In patients with biochemical androgen deficiency, 9(9.8\%) had diabetes, and 18 (19.6\%) had hypertension. These proportions were not significantly different compared with normal group $(P>0.05)$.

Biochemical tests were not significantly different in patients with cervical, thoracic and lumbosacral levels of SCI and between those with complete and incomplete injury $(P>0.05)$. Age and duration of injury were comparable in three different levels of SCI and complete versus incomplete groups.

Significant correlations between TT level and SHBG $(r=0.3$, $P<0.001)$, LH $(r=0.2, P=0.02), \mathrm{TC}(r=-0.1, P=0.04)$ and TG $(r=-0.3, P<0.001)$ were found.

Association of independent variables with risk of biochemical androgen deficiency in univariate and multivariate logistic regression analysis are summarized in Table 3 . Men who reported opioid use in their medical history had significantly higher odds of having biochemical androgen deficiency in comparison to the rest of the sample $(\mathrm{OR}=2.02,95 \% \mathrm{CI}$ : $1.01-4.09, P=0.042)$; however, opioid use was not significant in multivariate model.

\section{DISCUSSION}

SCI in adult men may result in various hormonal changes including testosterone deficiency. ${ }^{10}$ Normal physiological concentrations of testosterone may affect the overall health of men. Decrease in serum testosterone levels is associated with several complications. These problems seem to have a negative impact on men's self-confidence, quality of life and well-being. ${ }^{5}$

In current study the overall prevalence of biochemical androgen deficiency was $29.1 \%$ among all participants with the mean age of 47 years, which is higher than the prevalence of this problem in healthy men and in patients with ED. The Boston Area Community Health $(\mathrm{BACH})$ survey demonstrated low total $\left(<300 \mathrm{ng} \mathrm{dl}^{-1}\right)$ and free $\left(<5 \mathrm{ng} \mathrm{dl}^{-1}\right)$ testosterone plus one specific symptom (for example, ED) or two nonspecific symptoms in $5.6 \%$ of their studied population with the mean age of 47.3 years. ${ }^{18}$

In another study, the prevalence of androgen deficiency in Taiwanese men (mean age $=57.4$ years) was $24.1 \%$ when TT level $<300 \mathrm{ng} \mathrm{dl}^{-1}$ was considered as the criterion, and $16.6 \%$ considering both TT $<300 \mathrm{ng} \mathrm{dl}^{-1}$ and FT $<5 \mathrm{ng} \mathrm{dl}^{-1}$ as the criteria. ${ }^{19}$
Testosterone deficiency in non-SCI men with ED was reported 26.6 and $16.9 \%$ in the studies conducted by Somani et al. ${ }^{20}$ and Corona et al., ${ }^{21}$ respectively.

In the two most recent studies, the reported prevalence of low testosterone in men with chronic SCI was close to the findings in present study. In a large series (243 men), Bauman et al. ${ }^{6}$ reported low TT levels in $46 \%$ of their subjects considering a cutoff of $11.3 \mathrm{nmoll}^{-1}$; however, when the cutoff of $10 \mathrm{nmoll}^{-1}$ was considered, the prevalence of low TT dropped to $27 \%$. The mean age of participants was similar to that in the present study (48 years). In the report by Barbonetti et al., ${ }^{22}$ the prevalence of biochemical androgen deficiency $\left(\mathrm{TT}<300 \mathrm{ng} \mathrm{dl}^{-1}\right)$ was $35.3 \%$ among their participants with the mean age of 46.7 years. In this study, the prevalence of severe biochemical androgen deficiency $\left(\mathrm{TT}<230 \mathrm{ng} \mathrm{dl}^{-1}\right.$ with FT $\left.<63.4 \mathrm{pg} \mathrm{ml}^{-1}\left(220 \mathrm{pmoll}^{-1}\right)\right)$ was $25.5 \%$. The remaining patients (TT between 230 and $300 \mathrm{ng} \mathrm{dl}^{-1}$ ) had normal levels of FT.

Sex hormone binding globulin abnormality could affect the diagnosis of androgen deficiency based on TT level. In this condition, measurement of FT level is recommended. ${ }^{7}$ Accordingly, the high prevalence $(43.3 \%)$ of testosterone deficiency in men with chronic SCI reported by Durga et al. ${ }^{10}$ was probably related to the lack of data on SHBG and FT levels. In our studied population, SHBG abnormality was $15 \%$.

So far several studies have investigated serum testosterone and gonadotropins status in men with SCI; however, there have been some inconsistencies with regard to some differences in study methods. One major difference is heterogeneity of time since injury in studies. Clark et al. ${ }^{11}$ found high prevalence of low serum TT levels (60\%) in men with SCI, while $66 \%$ of their subjects were in acute phase. Decline in testosterone levels in acute phase of SCI could be related to the acute physical shock, ${ }^{9}$ as Celik et al. ${ }^{23}$ demonstrated that a negative androgen status is not notable after the first year of SCI. In our study, all participants were in chronic phase of SCI and there was no association between time since injury and hormonal values. Durga et al. ${ }^{10}$ and safarinejad ${ }^{24}$ were also not able to find any significant association between these variables in men with chronic SCI.

The neurological level and completeness of injury were not significantly different in biochemical androgen deficient and normal groups. In addition, these injury variables did not affect other hormonal profiles. The association of injury variables and alterations in testosterone and gonadotropins secretion were inconsistent in previous studies. ${ }^{6,10,11,24}$ Using different methods for determining neurological level and extent of injury may result in these 
Table 3 Association of independent variables with the risk of biochemical androgen deficiency in logistic regression analysis

\begin{tabular}{|c|c|c|c|c|}
\hline \multirow[t]{2}{*}{ Variables } & \multicolumn{2}{|c|}{ Model $1^{\mathrm{a}}$} & \multicolumn{2}{|c|}{ Model $2^{\mathrm{b}}$} \\
\hline & OR $(95 \% \mathrm{Cl})$ & P-value & OR $(95 \% \mathrm{Cl})$ & P-value \\
\hline Duration (year) & $1.04(0.99-1.09)$ & 0.095 & $1.04(0.98-1.11)$ & 0.115 \\
\hline Severity (incomplete/complete) & $1.35(0.82-2.21)$ & 0.236 & $1.19(0.69-2.05)$ & 0.510 \\
\hline \multicolumn{5}{|l|}{ SCI.L } \\
\hline Lumbosacral & $1.56(0.68-3.56)$ & 0.290 & $1.34(0.55-3.26)$ & 0.505 \\
\hline Smoking (yes/no) & $1.54(0.91-2.61)$ & 0.106 & $1.33(0.74-2.39)$ & 0.324 \\
\hline Opioid use (yes/no) & $2.02(1.01-4.09)$ & 0.048 & $1.57(0.72-3.41)$ & 0.251 \\
\hline
\end{tabular}

Abbreviations: $\mathrm{Cl}$, confidence interval; OR, odds ratio; SCI.L, spinal cord injury level.

a Model $1=$ univariate model.

bModel 2 = multivariate model (with adjustment). Copyright: 123 .

inconsistencies. Our results are in line with those of Barbonetti et al., ${ }^{22}$ using the same protocol to define both level and completeness of the lesion.

Dyslipidemia is prevalent in patients with SCI. ${ }^{25}$ Testosterone has a key role in lipid metabolism. Indeed, testosterone deficiency is related to elevated TG and TC. In current study, biochemical androgen deficient patients had higher TG and TC levels. This suggests that, testosterone replacement therapy may be beneficial in improving the lipid profile in SCI patients with low serum testosterone level. ${ }^{26}$

Taking opioids has not been considered in most comparable studies, ${ }^{6,11,24}$ although it could affect testosterone levels through suppressing the hypothalamic- pituitary- gonadal axis and the secretion of gonadotropin-releasing hormone. ${ }^{27,28}$ Chronic pain is one of the common problems in many patients with $\mathrm{SCI},{ }^{29}$ and a considerable number of these patients take opioids for pain relief. ${ }^{10}$ Some studies have indicated that using long-acting opioids result in androgen deficiency, ${ }^{7}$ while others have demonstrated that suppression in testosterone production happens in men taking opioids regularly, regardless of opioid type. ${ }^{30}$ In spite of prescribing short-acting opioid analgesics on an as needed basis in our studied population, opioid usage was associated with biochemical androgen deficiency in our SCI patients. Although opioid use was statistically significant in univariate regression, it was not significant in multivariate regression. This indicates that the effect of opioid is probably dependent on other variables in our participants. These data could be useful and worthy of further exploration.

Finally it would be appropriate to mention our study limitations. First, having a retrospective design, our study had some missing data such as body mass index. The second limitation is the lack of a non-SCI matched control group. Despite these limitations, this study had a larger sample size in comparison with other literatures evaluating androgen deficiency in spinal cord injured men. In addition, in relation to low serum testosterone level, we included as many possible factors as our study design permitted.

In conclusion, a substantial proportion of our patients with chronic SCI and ED had biochemical androgen deficiency. This proportion is comparable to recent studies' outcomes on SCI patients. Moreover, opioid use, TG and TC levels were associated with biochemical androgen deficiency in our studied population. Standard screening of androgen deficiency and testosterone replacement therapy are recommended in men with chronic SCI suffering from ED.

\section{DATA ARCHIVING}

There were no data to deposit.

\section{CONFLICT OF INTEREST}

The authors declare no conflict of interest.

\section{ACKNOWLEDGEMENTS}

We wish to thank PH Kolivand for general management in Khatam Al-Anbia Hospital and Dr S Azad-Barmaki and B Aslanbeigi for their supervision at the laboratory assignment. This study was supported by Tehran University of Medical Sciences (TUMS).

1 Schopp LH, Clark M, Mazurek MO, Hagglund KJ, Acuff ME, Sherman AK et al. Testosterone levels among men with spinal cord injury admitted to inpatient rehabilitation. Am J Phys Med Rehabil 2006; 85: 678-684.

2 Bauman WA, Spungen AM, Adkins RH, Kemp BJ. Metabolic and endocrine changes in persons aging with spinal cord injury. Assist Technol 1999; 11: 88-96.

3 Ricciardi R, Szabo CM, Poullos AY. Sexuality and spinal cord injury. Nurs Clin North Am 2007; 42: 675-684.

4 Myers J, Lee M, Kiratli J. Cardiovascular disease in spinal cord injury: an overview of prevalence, risk, evaluation, and management. Am J Phys Med Rehabil 2007; 86: $142-152$.

5 Mulligan T, Frick MF, Zuraw QC, Stemhagen A, Mcwhirter C. Prevalence of hypogonadism in males aged at least 45 years: the HIM study. Int J Clin Pract 2006; 60: 762-769.

6 Bauman WA, La Fountaine MF, Spungen AM. Age-related prevalence of low testosterone in men with spinal cord injury. J Spinal Cord Med 2014; 37: 32-39.

7 Bhasin S, Cunningham GR, Hayes FJ, Matsumoto AM, Snyder PJ, Swerdloff RS et al. Testosterone therapy in men with androgen deficiency syndromes: an endocrine society clinical practice guideline. J Clin Endocrinol Metab 2010; 95: 2536-2559.

8 Fourie T. The incidence of testosterone deficiency in men with long-term spinal cord injury. J Mens Health 2009; 6: 191-195.

9 Kalyani RR, Gavini S, Dobs AS. Male hypogonadism in systemic disease. Endocrinol Metab Clin North Am 2007; 36: 333-348.

10 Durga A, Sepahpanah F, Regozzi M, Hastings J, Crane DA. Prevalence of testosterone deficiency after spinal cord injury. PM R 2011; 3: 929-932.

11 Clark MJ, Schopp LH, Mazurek MO, Zaniletti I, Lammy AB, Martin TA et al. Testosterone levels among men with spinal cord injury: relationship between time since injury and laboratory values. Am J Phys Med Rehabil 2008; 87: 758-767.

12 Maynard FM Jr, Bracken MB, Creasey G, Ditunno JF Jr, Donovan WH, Ducker TB et al. International standards for neurological and functional classification of spinal cord injury. Spinal Cord 1997; 35: 266-274.

13 Rosen RC, Riley A, Wagner G, Osterloh IH, Kirkpatrick J, Mishra A. The International Index of Erectile Function (IIEF): a multidimensional scale for assessment of erectile dysfunction. Urology 1997; 49: 822-830.

14 Wang C, Nieschlag E, Swerdloff R, Behre HM, Hellstrom WJ, Gooren LJ et al. Investigation, treatment and monitoring of late-onset hypogonadism in males: ISA, ISSAM, EAU, EAA and ASA recommendations. Eur J Endocrinol 2008; 159: 507-514.

15 Vermeulen A, Verdonck L, Kaufman JM. A critical evaluation of simple methods for the estimation of free testosterone in serum. J Clin Endocrinol Metab 1999; 84: 3666-3672. 
16 Melmed S, Casanueva FF, Hoffman AR, Kleinberg DL, Montori VM, Schlechte JA et al. Diagnosis and treatment of hyperprolactinemia: an Endocrine Society clinical practice guideline. J Clin Endocrinol Metab 2011; 96: 273-288.

17 Casanueva FF, Molitch ME, Schlechte JA, Abs R, Bonert V, Bronstein MD et al. Guidelines of the Pituitary Society for the diagnosis and management of prolactinomas. Clin Endocrinol (Oxf) 2006; 65: 265-273.

18 Araujo AB, Esche GR, Kupelian V, O'Donnell AB, Travison TG, Williams RE et al. Prevalence of symptomatic androgen deficiency in men. J Clin Endocrinol Metab 2007; 92: 4241-4247.

19 Liu CC, Wu WJ, Lee YC, Wang CJ, Ke HL, Li WM et al. The prevalence of and risk factors for androgen deficiency in aging Taiwanese men. J Sex Med 2009; 6: 936-946.

20 Somani B, Khan S, Donat R. Screening for metabolic syndrome and testosterone deficiency in patients with erectile dysfunction: results from the first UK prospective study. BJU Int 2010; 106: 688-690.

21 Corona G, Mannucci E, Petrone L, Balercia G, Paggi F, Fisher AD et al. NCEP-ATPIII defined metabolic syndrome, type 2 diabetes mellitus, and prevalence of hypogonadism in male patients with sexual dysfunction. J Sex Med 2007; 4: 1038-1045.

22 Barbonetti A, Vassallo MR, Pacca F, Cavallo F, Costanzo M, Felzani G et al. Correlates of low testosterone in men with chronic spinal cord injury. Andrology 2014; 2: 721-728.
23 Celik B, Sahin A, Caglar N, Erhan B, Gunduz B, Gultekin O et al. Sex hormone levels and functional outcomes: a controlled study of patients with spinal cord injury compared with healthy subjects. Am J Phys Med Rehabil 2007; 86: 784-790.

24 Safarinejad MR. Level of injury and hormone profiles in spinal cord-injured men. Urology 2001; 58: 671-676.

25 Flank P, Ramnemark A, Levi R. Dyslipidemia is common after spinal cord injuryindependent of clinical measures. J Phys Rehabil Med 2015; 1: 001.

26 Kelly DM, Jones TH. Testosterone: a metabolic hormone in health and disease. J Endocrinol 2013; 217: 25-45.

27 Reddy RG, Aung T, Karavitaki N, Wass JAH. Opioid induced hypogonadism. BMJ 2010; 341: $c 4462$.

28 Katz N, Mazer NA. The impact of opioids on the endocrine system. Clin J Pain 2009; 25: $170-175$.

29 Cardenas DD, Bryce TN, Shem K, Richards JS, Elhefni H. Gender and minority differences in the pain experience of people with spinal cord injury. Arch Phys Med Rehabil 2004; 85: 1774-1781.

30 Bawor M, Bami H, Dennis BB, Plater C, Worster A, Varenbut M et al. Testosterone suppression in opioid users: a systematic review and meta-analysis. Drug Alcohol Depend 2015; 149: 1-9. 